\title{
Antibacterial Activity of Curcuma longa (turmeric), Curcuma zedoaria (zedoary), and Allium sativum (garlic) Nanoparticle Extract on Chicken with Chronic Respiratory Disease Complex: In Vivo Study
}

\author{
Ekowati Handharyani ${ }^{1 *}$, Lina N. Sutardi ${ }^{2}$, Aulia A. Mustika ${ }^{3}$, Andriani Andriani ${ }^{4}$, and Sri Yuliani $^{5}$ \\ ${ }^{1}$ Division of Pathology, Department of Clinic, Reproduction, and Pathology, IPB University, Bogor Indonesia \\ ${ }^{2}$ Division of Veterinary Pharmacy Department of Clinic, Reproduction, and Pathology, IPB University, Bogor Indonesia \\ ${ }^{3}$ Division of Pharmacology, Department of Anatomy, Physiology, and Pharmacology, IPB University, Bogor Indonesia \\ ${ }^{4}$ Indonesian Research Center for Veterinary Science, Bogor, Indonesia \\ ${ }^{5}$ Center for Post-harvest Agriculture Research and Development, Bogor Indonesia
}

\begin{abstract}
Previous in vitro studies showed that nanoparticle extract of turmeric, zedoary, and garlic exhibit antibacterial activity against Mycoplasma gallisepticum (M. gallisepticum) which causes chronic respiratory disease (CRD) in chicken. This research aimed to determine the antibacterial activity of nanoparticles of Curcuma longa, Curcuma zedoaria, and Allium sativum extract to CRD infected chicken. In vivo test of antibacterial activity of turmeric, zedoary, and garlic nanoparticle in combination was conducted on chicken infected by M. gallisepticum and Escherichia coli (E.coli). Antibiotic control used was enrofloxacin. As many as 75 chickens were divided into 5 groups containing 15 chickens each. Group one consisted of healthy chickens (positive control); group two consisted of chickens that have been inoculated by bacteria (negative control); group three (treatment) were chickens inoculated by bacterium and given extract nanoparticle combination on day 7 of infection for 7 days; group four (prevention) were chickens inoculated by bacterium and given combination of extract nanoparticles on day 5 before infection for 14 days; group five were chickens inoculated with bacterium and given enrofloxacin antibiotics for 7 days. In vivo research results showed increased body weight and performance indicated by improvements in clinical signs, and gross pathology changes. The combination of three extract nanoparticles showed the best activity in controlling CRD in chicken, both as preventive and curative means.
\end{abstract}

Keywords: chronic respiratory disease, turmeric, wild ginger, garlic.

\section{Introduction}

Chronic respiratory diseases (CRD) is an endemic disease in chickens that cause huge economic loss in the poultry industry [1,2]. This disease is caused by $M$. gallisepticum. This bacterium is an important pathogen that can damage the economy of the commercial poultry industry. CRD complex treatment usually involves the use of antibiotics. Resistance towards several antibiotics by M. gallisepticum has been reported [3]. Information about the danger of resistance and antibiotic residue in livestock products prompt the need to find an alternative solution. The use of medicinal herbs is among the feasible alternative. Curing and preventing via the use of herbal medicine has several advantages: it is relatively safe for consumption, low toxicity, and has not been reported to leave residues.

Turmeric (Curcuma domestica) is known to act as an antioxidant [6,7], antibacterial $[4,5]$, antifungal $[7,8]$ and antivirus [9.10]. Zedoary (Curcuma zedoaria) can neutralize toxins, elevate joint pain, lower cholesterol levels [15] has antibacterial effects [13], antioxidant [11], and anti-inflammatory properties [12,14]. Garlic (Allium sativum) contains allicin which is a bioactive compound that has antibacterial effects. Other bioactive components are diallyl disulfide and diallyl trisulfide which also exhibit antibacterial [17,18], antitubercular [16], and antiprotozoal activities [19].

The use of plant extract as therapeutic and preventive treatment often have issues such as low water solubility as well as sensitivity to heat, light, metal ion, enzyme, and oxygen. Based on pharmacological effects and its characteristics, the plant extract is a promising candidate for new drug main ingredients such as a nanoparticle, nano-emulsion, and nanoencapsulation. The nanotechnology-based pharmaceutical form can solve formulation problems and improve the pharmacology effect. Nanoparticle size ranges between $10-1000 \mathrm{~nm}$. The nanoparticle has a large surface area and a large number of atoms by its surface and thus having low surface energy and surface tension. This eases particles to pass through cell membranes. These characteristics can be modified by

* Corresponding author: ekowatieko@apps.ipb.ac.id. 
controlling the size of the material, the chemical composition, modifying the surface, and controlling the interaction between particles [20]. Previous researches have proven that nanoparticle extract formulation can improve medicinal plant pharmaceutical activity. This research aimed to determine the efficacy of nanoparticle combination of turmeric, zedoary, and garlic extract in chicken suffering from chronic respiratory disease.

\section{Materials and methods}

\subsection{Extraction}

Simplicia extraction used the maceration method for $3 \mathrm{x}$ 24 hours by pharmaceutical grade ethanol $96 \%$ solvent. The sample and the solvent ration were 1:10.

\subsection{Nanoparticle preparations}

Nanoparticle formation begins with the formation of nanoemulsion $0.5 \% \mathrm{w} / \mathrm{v}$. Nanoemulsion preparation production used the inversion method with the formula: extract, dimethyl sulfoxide, Tween 80, glycerol, and water. The nanoemulsion preparation was made by mixing every ingredient except water. Water is then added into the mixture and mixed with a stirrer on $700 \mathrm{rpm}$ for 15 minutes. The nanoemulsion was then spray dried. The morphology of nanoparticles was evaluated by scanning electron microscopy. Nanoparticle combination extract for turmeric, zedoary, and garlic are in 1:1:1 ratio.

\subsection{Antibiotic}

The antibiotic used was enrofloxacin. The animal model was given an oral solution with a $10 \%$ concentration according to the recommended dosage $(10 \mathrm{mg} / \mathrm{kg} \mathrm{BW})$ for 7 days.

\subsection{Bacterial Isolate}

Bacterial isolates $M$. gallisepticum and E. coli were obtained from The Indonesian Research Center for Veterinary Science. $M$. gallisepticum medium is a special medium consisting of PPLO broth and as fertilizer yeast extract was used. E. coli medium used were Mueller Hinton broth and Mueller Hinton agar. The bacterial dosage used was $5 \times 10^{8} \mathrm{CFU}$ for $M$. gallisepticum and $5 \times 10^{6}$ for $E$. coli. Infection was done by injecting the isolates via the intra-air sac route followed by the intranasal route. M. gallisepticum infection was done when the chicken reached 15 days old whereas $E$. coli infection was administered when the chicken was 18 days old [22].

\subsection{Research Design}

As many as 75 chickens were divided into 5 groups containing 15 chickens each. Group one consisted of healthy chickens (control); group two consisted of chickens with CRD complex infection; group three were chickens infected with CRD complex and treated with extract nanoparticle combination for 14 days; group four (prevention) were infected chickens and given extract nanoparticles combination of on day 5 before infection for
14 days; group five were chickens with CRD complex infection and given antibiotics for 7 days.

Nanoparticle dosage administration was based on the minimum inhibition dose for nanoparticle extract combination which was $2 \mathrm{mg} / \mathrm{kg} \mathrm{BW}$, with extract content being $0.4 \mathrm{mg} / \mathrm{kg} \mathrm{BW}$.

\subsection{Parameters}

The parameters for this research are clinical symptoms, body weight, feed conversion ratio, gross pathology, and histopathology. Serum samples were taken 1 day before infection, day 3 and 5 posts bacterial infection for SPA (Serum Plate Agglutination) test. Clinical symptoms and gross pathology were examined to determine the success of infection. Gross anatomy and histopathology features were gathered on days 22 and 29.

\section{Results and Discussion}

\subsection{Nanoparticle Preparation}

Particle size, distribution, and morphology are important factors in nanoparticle preparation. Size and distribution of nanoparticles can influence drug delivery, drug release, and nanoparticle stability. The scanning electron microscope (SEM) method was used to recognize the morphology of nanoparticles. The morphology of turmeric, zedoary, and garlic extract nanoparticle was found that had smooth surface and sphere shapes(Figure. $1)$.

(a)
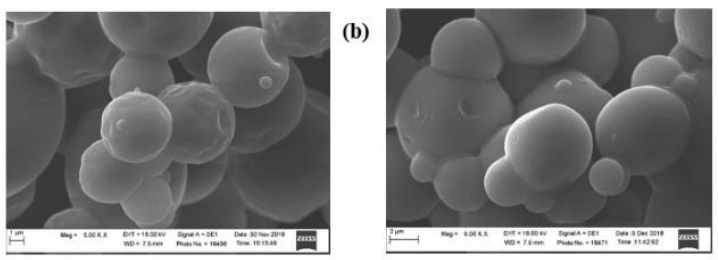

(c)

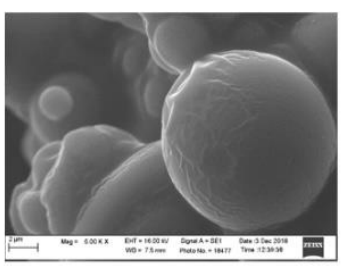

Figure. 1. Scanning Electron Microscope analysis (a) turmeric, (b) zedoary, and (c) garlic.
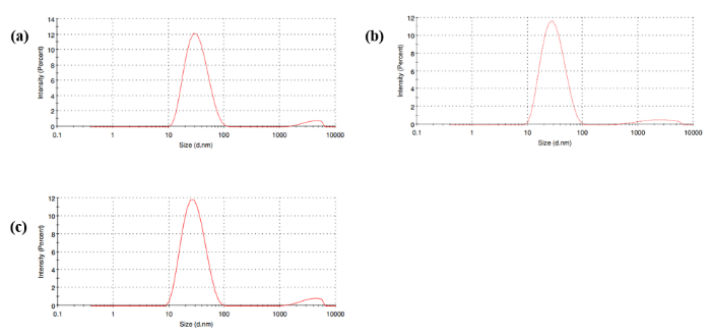

Figure. 2. Size distribution by intensity 
Particle size analysis results showed the average size of nanoparticles for turmeric extract was $30.60 \pm 0.85 \mathrm{~nm}$; zedoary was $28.33 \pm 0.87 \mathrm{~nm}$, and garlic was $26.86 \pm 0.83$ $\mathrm{nm}$. Particles of $200 \mathrm{~nm}$, and smaller, can penetrate cells much more easily than micron-sized particles eventually also passively [21]. The distribution of each nanoparticle is presented in Figure. 2. Aggregation of the nanoparticles usually cause an increase in the nanoparticle size [23].

\subsection{In vivo test}

Clinical symptoms began to be observed on day 7 postinfection (PI), characterized by lethargy, lowered appetite, ruffled feathers, nasal discharge, and snoring during breathing. On Group 4 (preventive) no clinical symptoms were observed. This condition may be caused by the effect of extract nanoparticle combination which can prevent the chickens from CRD manifestation.

Bodyweight measurement on therapeutic groups (extract treatment and antibiotic treatment) demonstrated similar body weight and body weight gain compare to the control group. Significant differences were observed between the therapeutic group and the infection group. Group 4 (preventive) has the best bodyweight and body weight gain (1550.7 $\pm 12.3 \mathrm{~g}$ and $64.18 \pm 2.6 \mathrm{~g}$, Table 1).

Feed conversion ratio (FCR) is among the determining indicator of feed efficiency rate in broiler chickens. Low FCR value indicates high feed efficiency. Infected broilers given nanoparticle extract combination formulation showed lowered values compared to the infected group without therapy (Group 2) which was significantly different $(\mathrm{P}<0,05)$. Group 3 (curative group) and group 4 (preventive group) have the best FCR rate, $1.71 \pm 0.04$ and $1.71 \pm 0.04$ (Table 1 ).

Table 1 Observation results of body weight, body weight gain, and FCR on day-29

\begin{tabular}{cccccc}
\hline Para- & \multicolumn{5}{c}{ Groups } \\
\cline { 2 - 6 } meter & 1 & 2 & 3 & 4 & 5 \\
\hline Body- & $1498.3 \pm$ & 1356.0 & $1458.0 \pm$ & $1550.7 \pm$ & 1509.7 \\
weight & $129.1^{\mathrm{ab}}$ & $\pm 40.6^{\mathrm{b}}$ & $39.2^{\mathrm{ab}}$ & $12.3^{\mathrm{a}}$ & $\pm 19.6^{\mathrm{ab}}$ \\
& & & & & \\
Body- & $6.47 \pm$ & $53.46 \pm$ & $62.73 \pm$ & $64.18 \pm$ & $63.25 \pm$ \\
weight & $0.02^{\mathrm{a}}$ & $1.31^{\mathrm{b}}$ & $1.02^{\mathrm{a}}$ & $2.60^{\mathrm{a}}$ & $2.45^{\mathrm{a}}$ \\
increase & & & & & \\
FCR & $1.73 \pm$ & $1.95 \pm$ & $1.71 \pm$ & $1.71 \pm$ & $1.77 \pm$ \\
& $0.02^{\mathrm{b}}$ & $0.05^{\mathrm{a}}$ & $0.04^{\mathrm{bc}}$ & $0.03^{\mathrm{bc}}$ & $0.02^{\mathrm{b}}$ \\
& & & & & \\
\hline
\end{tabular}

Rising body weight and its increase after being given nanoparticle extract combination formulae are assumed to have been caused by the active compound within the nanoparticle material. Curcumin is an active compound contained in turmeric. This compound has the role of increasing the activity of gastrointestinal tracts, especially bile and pancreas. This activity can induce increases in production and enzyme secretion of gastrointestinal tracts to optimize the metabolism of carbohydrate, protein, and lipid source feed [27].

\subsection{Gross Pathology result of in vivo test}

Table 2 showed the image of gross pathology for each group during the research period. Pericarditis (40\%), airsacculitis $(100 \%)$, and perihepatitis $(20 \%)$ are the main lesions found in the infected group (group 2) on day 22. All treatment groups (extract combination and enrofloxacin) showed lower lesion percentage compared to group 2 (Table 2). On day 29, the chickens recovered from infection and lesions appeared to decrease, however, group 2 still showed more lesions compared to other groups with pericarditis (20\%) and airsacculitis (80\%). This proved that the administration of nanoparticle extract combination of turmeric, zedoary, and garlic can lower the incidence of perihepatitis, pericarditis, and airsacculitis similar to what was found in enrofloxacin group (Group 5).

Table 2 Observation result of chicken pathology anatomy

\begin{tabular}{|c|c|c|c|c|c|c|}
\hline \multirow{3}{*}{ Day } & \multirow{3}{*}{ Lesion } & \multicolumn{5}{|c|}{ Total number of cases $(\%)$} \\
\hline & & \multicolumn{5}{|c|}{ Groups } \\
\hline & & 1 & 2 & 3 & 4 & 5 \\
\hline \multirow{3}{*}{22} & Perihepatitis & 0 & 20 & 100 & 20 & 100 \\
\hline & Pericarditis & 0 & 40 & 0 & 0 & 0 \\
\hline & Airsacculitis & 0 & 100 & 100 & 60 & 100 \\
\hline \multirow{3}{*}{29} & Perihepatitis & 0 & 0 & 0 & 0 & 0 \\
\hline & Pericarditis & 0 & 20 & 0 & 0 & 0 \\
\hline & Airsacculitis & 0 & 80 & 0 & 0 & 0 \\
\hline
\end{tabular}

\subsection{Lung Histopathology}

Chronic Respiratory Disease case in chicken has an infection mechanism. M. gallisepticum bacteria enters the nasal cavity and attaches to epithelial receptor called sialoglycoprotein (Patron recognition receptors sites). This attachment is mediated by adhesin and protein called bleb (pathogen associate molecular patrons) located on the tip of mycoplasma bacteria's organ. Mycoplasma penetrates and destroys epithelial mucosa while multiplying itself with the help of epithelial cilia and bleb. Mycoplasma moves into the abdominal air sac. $M$. gallisepticum then moves into the ovary or oviduct to cause vertical transmission in which the complete mechanism is still unknown [24]. Lung histopathology is presented on Figure 3.

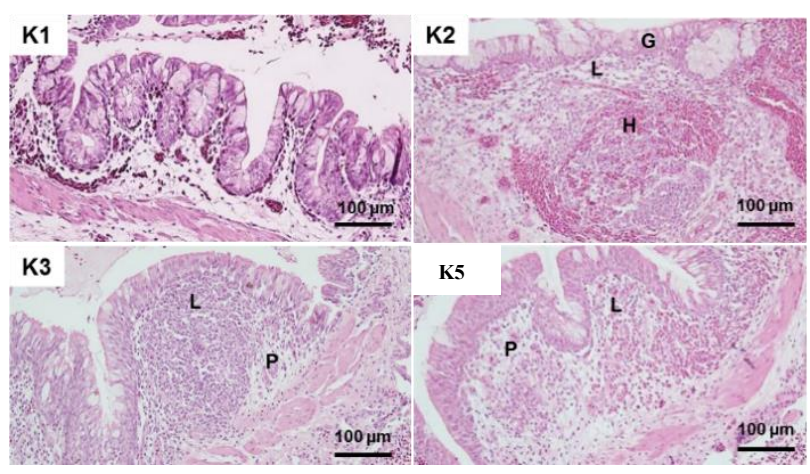

Figure 3. Lung histopathology around secondary bronchi; K1 is a healthy condition of the chicken lung. $\mathrm{K} 2$ is the lung of infected chicken showed lymphocytes infiltration (L), Goblet cell degeneration $(\mathrm{G})$ and hemorrhage $(\mathrm{H})$. K3 (preventive) and 
K5 (antibiotic treatment) showed lung with lymphocyte aggregates $(\mathrm{L})$ with the recovery process $(\mathrm{P})$. HE staining.

Histopathology evaluation results of chicken lungs showed pathological changes of three groups, which are bacterial infected (K2), administered extract nanoparticle combination (K3), and antibiotic (K5). The three groups showed changes in the chicken lung around the bronchioli. Histopathological examination on $\mathrm{K} 1$ showed that the lung did not show any changes. K2 (infected chickens) showed infection surrounding bronchioli, indicated by inflammatory cells (lymphocyte) mixed with hemorrhage at the bronchus associated lymphoid tissue (BALT) area. Goblet cells on the mucosa showed degeneration. Chicken infected by the bacteria and administered nanoparticle extract combination (K3) showed an increase in the number of lymphocyte cells by the BALT area and the number of BALT. This condition explains the microscopically increased readiness of lymphocyte cells in the BALT area to occur, according to Reese et al. (2006). There is an assumption that nanoparticle extract combination given to the $\mathrm{K} 3$ group act as an immunomodulator [25,26]. Histopathological image of K5 showed a lowered number of lymphocytes in the BALT area compared to K3. Recovery in K3 and K5 group is assumed to be an effect of nanoparticle herbal materials administration. Recovery criteria from nanoparticle extract combination and antibiotic are lowered number of lesions (hemorrhage and inflammatory cell infiltration) caused by CRD complex.

\section{Conclusion}

The in vivo study of nanoparticle extract combination using turmeric, zedoary, and garlic showed improvement in growth and performance with recovery in clinical and pathological changes of CRD complex infection. Its combination has both preventive and curative activities.

\section{Acknowledgments}

We would like to express gratitude to the Agricultural Research and Development Agency Ministry of Agriculture for funding this research through KP4S Research Program Fiscal Year 2017. We would also thank the Dean of Veterinary Faculty IPB University, the Head of Indonesian Research Center for Veterinary Science and Bogor Agricultural Post-harvest Research and Development Center in Bogor; for the facilities, they had provided. Authors declare that there is no conflict of interest with parties involved in this research.

\section{References}

1. Mohammed HO, Tim EC, Yamamoto R: Economic Impact of Mycoplasma gallisepticum and M. synoviae in Commercial Layer Flocks. Avian Dis. 1987;31(3):477482 .

2. Gharaibeh S, Alaa H: Mycoplasma gallisepticum experimental infection and tissue distribution in chickens, sparrows, and pigeons. Avian Pathol. 2011 40(4):349-354.
3. Zanella A, Martino PA, Pratelli A et al.: Development of antibiotic resistance in Mycoplasma gallisepticum in vitro. Avian Pathol. 1998;27(6):591-596.

4. Singh D, Vandana R, Shivaraj N et al.: Biosynthesis of silver nanoparticle by endophytic fungi Pencillium sp. isolated from Curcuma longa (turmeric) and its antibacterial activity against pathogenic gram negative bacteria. J Pharm Res. 2013; 7:448-453.

5. Hafida FH, Dalila B, Tayeb B: Hydrogel nanocomposites based on chitosan-gpolyacrylamide and silver nanoparticles synthesized using Curcuma longa for antibacterial applications. Polym Bull 2018; 75:2819-2846.

6. Eric WCC, Voon PN, Vi VT et al.: Antioxidant and Antibacterial Properties of Alpinia galanga, Curcuma longa, and Etlingera elatior (Zingiberaceae). Ph⿻og J. 2011;3(22):54-61.

7. Bhanu P, Priyanka A, Akash K et al.: Efficacy of essential oil combination of Curcuma longa 1 . and Zingiber officinale rosc. as a postharvest fungitoxicant, aflatoxin inhibitor and antioxidant agent. J Food Saf. 2012;1-10.

8. Yichen H, Jinming ZC, Weijun K, et al:: Mechanisms of antifungal and anti-aflatoxigenic properties of essential oil derived from turmeric (Curcuma longa $\mathbf{L}$.) on Aspergillus flavus. Food chem. 2012; 220:1-8

9. Kima HJ, Hwa SY, Jin CK, et al.: Antiviral effect of Curcuma longa Linn extract against hepatitis B virus replication. J Ethnopharmacol. 2009; 124:189-196.

10. Ichsyani M, Ridhanya A, Risanti M et. al: : Antiviral effects of Curcuma longa $\mathrm{L}$. against dengue virus in vitro and in vivo. IOP Conf. Series: Earth and Environmental Science. 2017.

11. Pratibha S, Sunita S, Kapoora IPS et. al: Chemical composition and antioxidant activities of essential oil and oleoresins from Curcuma zedoaria rhizomes. Food Biosci. 2013; 3:42-48.

12. Makabe $\mathrm{H}$, Maru $\mathrm{N}$, Kuwabara $\mathrm{A}$ et al.: Antiinflammatory sesquiterpenes from Curcuma zedoaria. Nat Prod Res: Formerly Natural Product Letters. 2006; 20(7):680-685.

13. Eric YCL, Charng-Cherng $\mathrm{C}$, Jeng-Leun $\mathrm{M}$ et al.: Antimicrobial Activity and Cytotoxicity of the Essential Oil of Curcuma zedoaria. Am J Chin Med. 2004; 32(2):281-290.

14. Arif HMAU, Sayera Z, Fatematuj J et al.: Evaluation of antinociceptive, in-vivo \& in-vitro anti-inflammatory activity of ethanolic extract of Curcuma zedoaria rhizome. BMC Complement Altern Med. 2014; 14:346

15. Tariq: Phytopreventive antihypercholesterolmic and antilipidemic perspectives of zedoary (Curcuma Zedoaria Roscoe.) herbal tea. Lipids in Health Dis. 2016; 15:39

16. Gupta RL, Sundeep J, Talwar V et al.: Antitubercular activity of garlic (allium sativum) extract in combination with conventional antitubercular drugs in tubercular lymphadenitis. IJCB. 1999; 14(1):12-18.

17. Lori R, Arunachalam J: Sunlight based irradiation strategy for rapid green synthesis of highly stable silver nanoparticles using aqueous garlic (Allium sativum) extract and their antibacterial potential. Mater Chem and Phys. 2011; 129:558-563.

18. Bashir L, Oluwatosin KS, Florence IO, et al.: Antimicrobial evaluation, acute and sub-acute toxicity 
studies of Allium sativum. J Acute Dis. 2016; 5(4): 296301.

19. Mandira S, Bandyopadhyay PK: Phytochemical screening for identification of bioactive compound and antiprotozoan activity of fresh garlic bulb over trichodinid ciliates affecting ornamental goldfish. Aquaculture. 2017; 473:181-190.

20. Amrita B, Jianping Q, Rohan G, et al.: Role of Nanoparticle Size, Shape and Surface Chemistry in Oral Drug Delivery. J Controlled Release. 2016; 238:176-185.

21. Peter G: Interaction of nanoparticles with biological systems. Colloid Surfaces B. 2018; 172:395-399.

22. Xiao X, Zhao DH, Yang X: Mycoplasma gallisepticum and Escherichia coli mixed infection model in broiler chickens for studying valnemulin pharmacokinetics. J Vet Pharmacol Ter. 2013; 37:99102.
23. Zangeneh MM, Hori G, Mohsen A: Novel synthesis of Falcaria vulgaris leaf extract conjugated copper nanoparticles with potent cytotoxicity, antioxidant, antifungal, antibacterial, and cutaneous wound healing activities under in vitro and in vivo condition. J Photoch Photobio B. 2019; 197:1-13.

24. Soeripto: Chronic Respiratory Disease (CRD) pada Ayam. Wartazoa. 2009; 19(3):135-143.

25. Yadav VS, Mishra KP, Singh DP et al.: Immunomodulatory effect of curcumin. $J$ Immunopharmacol Immunotoxicol. 2005; 27:485-497.

26. Culdell DR, Wilkinson F: Curcumin: Powerful immunomodulator from turmeric. Current Immunol Rev. 2014; 10(2):1-11.

27. Rajasekaran SA: Therapeutic potential of curcumin in gastrointestinal diseases. World $J$ Gastrointest Pathophysiol. 2011; 2(1):1-14. 